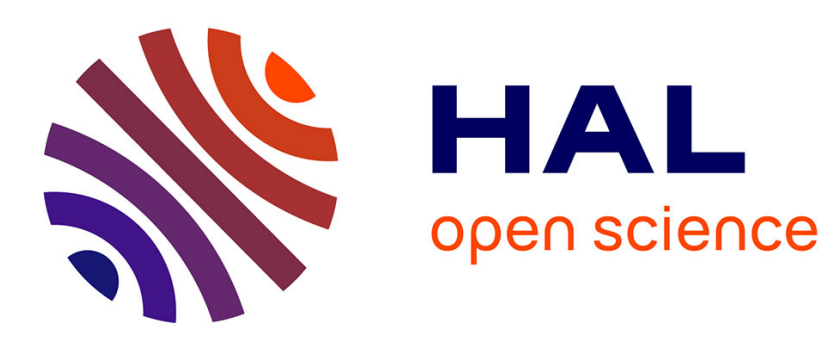

\title{
Direct measurement of the Wigner time delay for the scattering of light by a single atom
}

R Bourgain, J Pellegrino, Stephan Jennewein, Yvan R. P. Sortais, Antoine Browaeys

\section{- To cite this version:}

R Bourgain, J Pellegrino, Stephan Jennewein, Yvan R. P. Sortais, Antoine Browaeys. Direct measurement of the Wigner time delay for the scattering of light by a single atom. Optics Letters, 2013, 38, pp.1963-1965. 10.1364/OL.38.001963 . hal-01102237

\section{HAL Id: hal-01102237 \\ https://hal-iogs.archives-ouvertes.fr/hal-01102237}

Submitted on 12 Jan 2015

HAL is a multi-disciplinary open access archive for the deposit and dissemination of scientific research documents, whether they are published or not. The documents may come from teaching and research institutions in France or abroad, or from public or private research centers.
L'archive ouverte pluridisciplinaire HAL, est destinée au dépôt et à la diffusion de documents scientifiques de niveau recherche, publiés ou non, émanant des établissements d'enseignement et de recherche français ou étrangers, des laboratoires publics ou privés. 


\title{
Direct measurement of the Wigner time delay for the scattering of light by a single atom
}

\author{
R. Bourgain, J. Pellegrino, S. Jennewein, Y. R. P. Sortais, ${ }^{*}$ and A. Browaeys \\ Laboratoire Charles Fabry, Institut d'Optique, CNRS, Univ Paris Sud, 2 Avenue Augustin Fresnel, 91127 Palaiseau cedex, France \\ *Corresponding author: yvan.sortais@institutoptique.fr
}

Received April 15, 2013; accepted April 17, 2013;

posted April 23, 2013 (Doc. ID 188661); published May 30, 2013

\begin{abstract}
We have implemented the Gedanken experiment of an individual atom scattering a wave packet of near-resonant light, and measured the associated Wigner time delay as a function of the frequency of the light. In our apparatus, the atom behaves as a two-level system and we have found delays as large as $42 \mathrm{~ns}$ at resonance, limited by the lifetime of the excited state. This delay is an important parameter in the problem of collective near-resonant scattering by an ensemble of interacting particles, which is encountered in many areas of physics. (C) 2013 Optical Society of America

OCIS codes: (290.5820) Scattering measurements; (350.4855) Optical tweezers or optical manipulation; (290.4210) Multiple scattering; (020.1670) Coherent optical effects.

http://dx.doi.org/10.1364/OL.38.001963
\end{abstract}

The scattering of an incident wave, whether classical or quantum, upon a single particle is not an instantaneous process. In 1955, E. P. Wigner showed that the time-delay associated to the elastic scattering of a wave upon a scatterer is the derivative of the phase shift acquired by the incident wave with respect to its energy [1]. Later, F. T. Smith pointed out that this so-called Wigner delay is the lifetime of a resonant state excited during the scattering [2], therefore being largest at resonance. Since its derivation, the Wigner delay has been used as an important parameter in the problem of near-resonant scattering in dense media, as it governs the transport of energy [3-7].

Scattering processes are common in many areas of physics, and therefore many systems are candidates for the measurement of this delay. However, in most systems it is expected to be very short, explaining why experimental demonstrations are scarce and have required involved techniques. In 1976, a delay in the $10^{-20} \mathrm{~s}$ range was measured in the elastic near-resonant scattering of protons on a target of carbon using interferences in the Bremsstrahlung radiation [8]. The advent of ultrashort pulse laser-based metrology made it possible to measure time delays associated to the scattering of light by condensed matter systems or by atomic vapors. Femtosecond (fs) laser techniques, for instance, allowed to measure delays of a few fs associated with the bouncing of light off a metallic surface in the vicinity of a plasmon resonance [9]. More recently, attosecond metrology led to the measurement of delays in the 10-100 attoseconds range in the photoemission from a surface [10] or from a vapor $[11,12]$. Yet, the Gedanken experiment imagined initially by Wigner has never been realized, i.e., the direct measurement of the time delay induced by a single scatterer upon an incident wave. Here, we do so by sending a Gaussian wavepacket of near-resonant light on an isolated individual atom and by scanning the frequency of the light across an atomic resonance.

The Wigner delay can be understood in a semiclassical model, where a two-level atom elastically scatters a classical light field. In the limit of weak intensities, the atom responds linearly to the incoming light field, and the associated dispersive behavior leads to a time delay of the re-emitted field that is maximum at resonance and is given by $\tau_{W}=d \phi / d \omega$. Here, $\omega$ is the frequency of light, $\phi(\omega)=\arctan \left(\Gamma / 2\left(\omega_{0}-\omega\right)\right)$ is the phase of the atomic polarizability $[13,14], \omega_{0}=2 \pi c / \lambda_{0}$ is the frequency of the atomic resonance, and $\Gamma$ is the inverse lifetime of the excited state $|e\rangle$ (see Fig. 1). $\tau_{W}$ is thus given by

$$
\tau_{W}(\omega)=\frac{2}{\Gamma} \frac{1}{1+4\left(\frac{\omega-\omega_{0}}{\Gamma}\right)^{2}} .
$$

When the incident wave is a weak pulse of light, the scattered field amplitude is obtained by adding coherently the scattered amplitudes associated to each Fourier component of the incident pulse, and $\tau_{W}$ is the delay in the arrival time of the pulse envelope, induced by the presence of the atom $[1,2, \underline{4}, 13]$. In the particular case of a Gaussian pulse of light with root-mean-square (rms) duration $\Delta t \gg 1 / \Gamma$ and small intensity $\left(I / I_{\text {sat }} \ll 1\right)$, the scattered pulse is undistorted, with an intensity given by

$$
I_{\mathrm{sc}}(r, t) \propto \frac{1}{1+4\left(\frac{\omega_{L}-\omega_{0}}{\Gamma}\right)^{2}} e^{-\left[t-\frac{r}{c}-\tau_{W}\left(\omega_{L}\right)\right]^{2} / 2 \Delta t^{2}}
$$

(a)

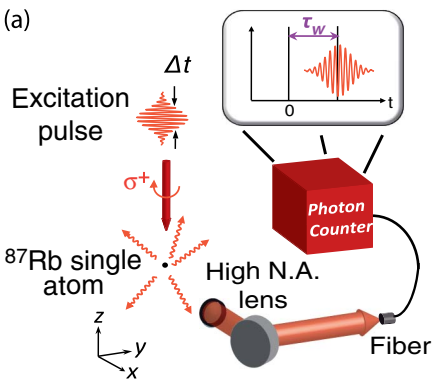

(b)

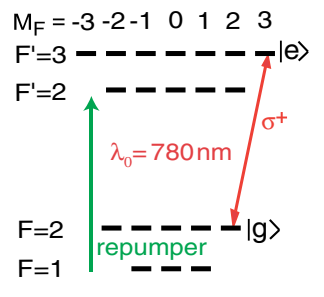

Fig. 1. (a) Implementation of the Wigner Gedanken experiment: we excite a single atom by a pulse of light and measure the arrival time of the scattered pulse on a photon counter; we use the large numerical aperture lenses to isolate a single atom with a tightly focused laser beam (not shown), and collect the scattered photons efficiently. (b) Atomic levels used in the experiment. 
Here, $\omega_{L}$ is the center frequency of the laser pulse spectrum, $t$ is time, $r$ is the distance between the observer and the scatterer, and $c$ is the velocity of light. In the limits mentioned above, we thus expect a scattered pulse that is Gaussian in temporal shape and maximally delayed at resonance by $\tau_{W}\left(\omega_{0}\right)=2 / \Gamma$, which can reach several tens of nanoseconds for optical transitions of e.g., alkali atoms.

To check this prediction experimentally, we use a single cold ${ }^{87} \mathrm{Rb}$ atom that we initially isolate in an optical dipole trap with microscopic size [15]. The temperature of the atom in the trap is $70 \mu \mathrm{K}$, measured by a releaseand-recapture method [16]. We first prepare the trapped atom in the hyperfine ground state level $\left(5 S_{1 / 2}, F=2\right)$. We then release it in free space and illuminate it with a series of weak Gaussian pulses of circularly polarized near-resonant laser light at $\lambda_{0}=780 \mathrm{~nm}$ (see Fig. 1). Using a large numerical aperture lens (N.A. $=0.5$ ), we collect the photons scattered at $90^{\circ}$ with respect to the direction of excitation and detect them with a fibercoupled avalanche photodiode (APD) operating in the single photon counting mode. The signal is sent to a counting card with a 256 ps resolution. The excitation light is produced from a continuous laser locked on the $\left(5 S_{1 / 2}, F=2\right)$ to $\left(5 P_{3 / 2}, F^{\prime}=3\right)$ transition. We chop this light into pulses with a Gaussian temporal shape using an acousto-optic modulator and a fast arbitrary waveform generator. We set the peak intensity $I$ of the pulse to $I / I_{\text {sat }}=0.1\left(I_{\text {sat }}=1.6 \mathrm{~mW} / \mathrm{cm}^{2}\right)$ to operate in the weak excitation limit. For the temporal width of the pulses, we choose $\Delta t=66 \mathrm{~ns}$ sufficiently large to approach the limit $\Delta t \gg 1 / \Gamma$ (here, $1 / \Gamma=26 \mathrm{~ns}$ ), and sufficiently small to determine with a good accuracy the temporal center of the scattered pulse, and thus the time delay $\tau_{W}$ [see Fig. 2(a)]. The acousto-optic modulator allows us to tune the center frequency $\omega_{L}$ of the laser light around the frequency $\omega_{0}$ of the closed transition between the states $|g\rangle=\left|5 S_{1 / 2}, F=2, M_{F}=2\right\rangle$ and $|e\rangle=\mid 5 P_{3 / 2}, F^{\prime}=3$, $\left.M_{F}^{\prime}=3\right\rangle$. The optical pumping of the atom in the Zeeman sublevel $|g\rangle$ is ensured by the first excitation pulses when the laser is on resonance with the atom. When the laser is tuned away from the resonance, the optical pumping is less efficient. However the Wigner delay is unaffected by the events when the atom does not cycle on the closed

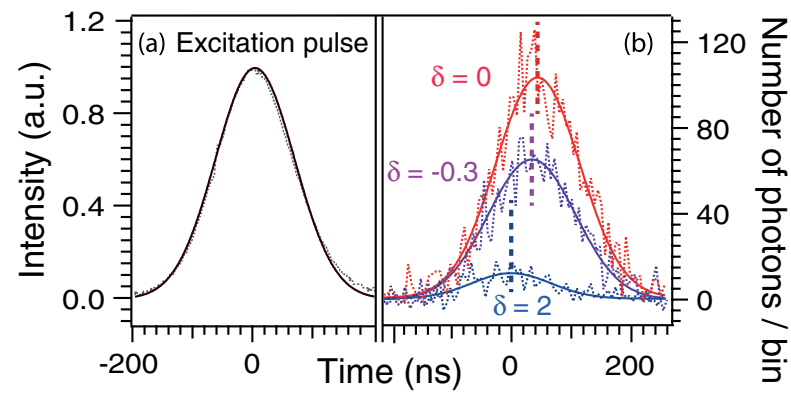

Fig. 2. (a) Intensity of the excitation pulse with rms width $\Delta t=66 \mathrm{~ns}$, (b) histograms of the number of photons detected after scattering for $\delta=\left(\omega_{L}-\omega_{0}\right) / \Gamma=(0 ;-0.3 ; 2)$ (time bins: $5.9 \mathrm{~ns})$. Dotted lines are the measured data and solid lines are Gaussian fits. In (b), the rms widths of the scattered pulses are, respectively, 73, 73, $65 \mathrm{~ns}$, in agreement with the solution of Eqs. (3). transition, as both the center frequency and the width of the resonance, which sets the value of the delay, are independent of the transition between the states $\left|5 S_{1 / 2}, F=2, M_{F}\right\rangle$ and $\left|5 P_{3 / 2}, F^{\prime}=3, M_{F}^{\prime}\right\rangle$. During the excitation, repumping light tuned to the $\left(5 S_{1 / 2}, F=1\right)$ to $\left(5 P_{3 / 2}, F^{\prime}=2\right)$ transition is also sent on the atom.

To maximize the number of collected photons scattered by the same atom, we use a time sequence where we interleave excitation pulses in free space with recapturing periods of $1.3 \mu \mathrm{s}$. After 50 such excitation-andrecapture periods, photon scattering has heated the atom and the probability that the atom escapes the trap has increased. We therefore apply a $1 \mathrm{~ms}$ period of three dimensional laser cooling with the dipole trap on. In this way, we keep the Doppler shift below $100 \mathrm{kHz}$. We repeat this pattern 120 times, corresponding to a total of 6000 pulses sent on the same atom before we start again with a newly prepared atom.

Figure 2(b) shows the temporal responses obtained on the photon counter for different values of the detuning $\delta=\left(\omega_{L}-\omega_{0}\right) / \Gamma$ of the excitation laser. Each temporal response results from an integration over 2000 individual atoms having experienced the sequence described above, and is fitted with a Gaussian to extract the arrival time of the scattered pulse. At resonance, the scattered pulse is maximally delayed (and most intense), as expected from Eqs. (1) and (2). Figure 3 summarizes the variation of the arrival time versus $\delta$. The comparison to far off-resonance measurements reveals a Wigner time delay $\tau_{W}$ as large as $42 \pm 2 \mathrm{~ns}$ at resonance, not far from the $2 / \Gamma=52$ ns predicted by Eq. (1).

The discrepancy is actually due to the conditions $\Delta t \gg 1 / \Gamma$ and $I / I_{\text {sat }} \ll 1$ not being exactly fulfilled experimentally. In particular, the latter condition means that the scattering is not only elastic, as implicit from the model discussed above, but has also a small inelastic component. To take this into account, we solved the optical Bloch equations governing the evolution of the density matrix for a two-level atom, using the measured shape of the excitation pulse as a driving term. These equations read in the rotating-wave approximation [17]:

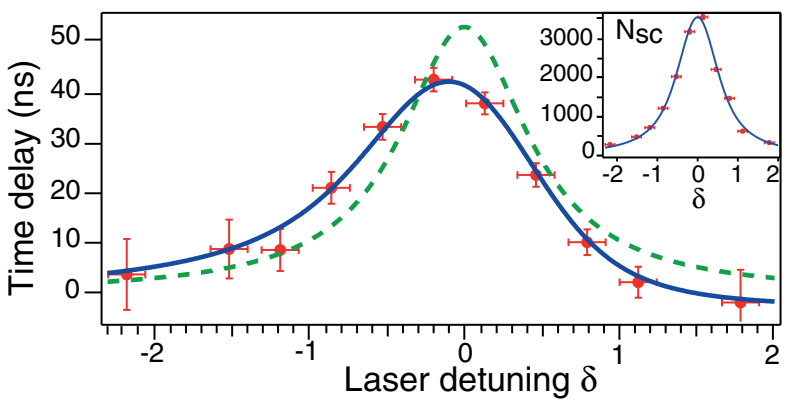

Fig. 3. Time delay of the scattered light pulse versus the detuning $\delta$ of the excitation laser. Dashed line: prediction of Eq. (1). Solid line: solution of Eqs. (3) for a two-level atom excited by the pulse shown in Fig. 2(a), with a fitted chirp rate $\alpha / 2 \pi=5 \pm 0.8 \mathrm{MHz} / \mu \mathrm{s}$. The vertical error bars are from the fits of the scattered pulses. Horizontal error bars are the rms uncertainty in the laser frequency $(0.12 \Gamma)$. The inset shows the number of scattered photons detected on the APD, and the solid line is the solution of Eqs. (3). 


$$
\begin{aligned}
& \dot{\tilde{\rho}}_{e g}=i\left(\omega_{L}-\omega_{0}(t)\right) \tilde{\rho}_{e g}+i \frac{\Omega(t)}{2}\left(2 \rho_{e e}-1\right)-\frac{\Gamma}{2} \tilde{\rho}_{e g}, \\
& \dot{\rho}_{e e}=i \frac{\Omega(t)}{2}\left(\tilde{\rho}_{e g}-\tilde{\rho}_{g e}\right)-\Gamma \rho_{e e}
\end{aligned}
$$

where $\tilde{\rho}_{e g}=\rho_{e g} \exp \left(i \omega_{L} t\right), \quad \tilde{\rho}_{e g}=\tilde{\rho}_{g e}^{*}, \quad \Omega(t)=\Omega e^{-t^{2} / 4 \Delta t^{2}}$ and $2 \Omega^{2} / \Gamma^{2}=I / I_{\text {sat }}$. In order to account for the slight asymmetry observed on the data (see Fig. 3), we have allowed for a small chirp of the transition frequency during the pulse: $\omega_{0}(t)=\omega_{0}+\alpha t$ [18]. The resolution of Eqs. (3) yields the temporal evolution of the population $\rho_{e e}(t)$ in state $|e\rangle$, proportional to the scattered pulse intensity $I_{\mathrm{sc}}(t)$. For our experimental parameters $I_{\mathrm{sc}}(t)$ is also Gaussian to a very good approximation, which allows us to identify unambiguously the Wigner delay [19]. A fit of the data by this second model with the chirp rate $\alpha$ as an adjustable parameter gives $\alpha / 2 \pi=$ $5 \pm 0.8 \mathrm{MHz} / \mu \mathrm{s}$ and predicts a maximum delay of $42 \mathrm{~ns}$ at resonance, in very good agreement with the measured delay. By setting $I / I_{\text {sat }}<0.01$ and $\Delta t>10 / \Gamma$ in Eqs. (3), as well as $\alpha=0$, we checked that we recovered the asymptotic value of the Wigner delay $2 / \Gamma$ characteristic of the elastic scattering regime to better than $2 \%$.

To characterize the scattering process fully we also analyze the number of collected photons as a function of the excitation detuning $\delta$. To do so, we integrate the temporal signal obtained on the APD. The data are again in good agreement with the solution of Eqs. (3), and exhibit a full width at half maximum of $1.2 \Gamma$ (see Fig. 3 inset), larger than the Lorentzian profile predicted by Eq. (2). This larger width is also consistent with the reduced time delay due to the conditions $\Delta t \gg 1 / \Gamma$ and $I / I_{\text {sat }} \ll 1$ not being exactly fulfilled experimentally.

In conclusion, we have measured the time delay introduced by an individual atom in an essentially elastic scattering process. We have found delays as large as $42 \mathrm{~ns}$, in good agreement with the theoretical limit predicted by the optical Bloch equations. In the future, it will be interesting to extend these measurements to the case of dense ensembles of cold interacting atoms to probe collective scattering [3] .

We acknowledge support from the E.U. through the ERC Starting Grant ARENA and the Integrated Project AQUTE, and from the Triangle de la Physique. We thank G. Labeyrie, T. Lahaye, A. Vernier and A. Aspect for useful discussions.

\section{References and Notes}

1. E. P. Wigner, Phys. Rev. 98, 145 (1955).

2. F. T. Smith, Phys. Rev. 118, 349 (1960).

3. A. Lagendijk and B. A. van Tiggelen, Phys. Rep. 270, 143 (1996).

4. C. A. A. de Carvalho and H. M. Nussenzveig, Phys. Rep. 364, 83 (2002).

5. G. Labeyrie, E. Vaujour, C. A. Müller, D. Delande, C. Miniatura, D. Wilkowski, and R. Kaiser, Phys. Rev. Lett. 91, 223904 (2003).

6. C. A. Müller, C. Miniatura, D. Wilkowski, R. Kaiser, and D. Delande, Phys. Rev. A 72, 053405 (2005).

7. R. Pierrat and R. Carminati, Phys. Rev. A 81, 063802 (2010).

8. C. Maroni, I. Massa, and G. Vannini, Phys. Lett. B 60, 344 (1976).

9. D. Chauvat, O. Emile, F. Bretenaker, and A. Le Floch, Phys. Rev. Lett. 84, 71 (2000).

10. A. L. Cavalieri, N. Müller, Th. Uphues, V. S. Yakovlev, A. Baltuška, B. Horvath, B. Schmidt, L. Blümel, R. Holzwarth, S. Hendel, M. Drescher, U. Kleineberg, P. M. Echenique, R. Kienberger, F. Krausz, and U. Heinzmann, Nature 449, 1029 (2007).

11. M. Schultze, M. Fieß, N. Karpowicz, J. Gagnon, M. Korbman, M. Hofstetter, S. Neppl, A. L. Cavalieri, Y. Komninos, Th. Mercouris, C. A. Nicolaides, R. Pazourek, S. Nagele, J. Feist, J. Burgdörfer, A. M. Azzeer, R. Ernstorfer, R. Kienberger, U. Kleineberg, E. Goulielmakis, F. Krausz, and V. S. Yakovlev, Science 328, 1658 (2010).

12. K. Klünder, J. M. Dahlström, M. Gisselbrecht, T. Fordell, M. Swoboda, D. Guénot, P. Johnsson, J. Caillat, J. Mauritsson, A. Maquet, R. Taïeb, and A. L'Huillier, Phys. Rev. Lett. 106, 143002 (2011).

13. J. D. Jackson, Classical Electrodynamics (Wiley, 1998).

14. L. Allen and J. H. Eberly, Optical Resonance and Two-Level Atoms (Dover, 1987).

15. Y. R. P. Sortais, H. Marion, C. Tuchendler, A. M. Lance, M. Lamare, P. Fournet, C. Armellin, R. Mercier, G. Messin, A. Browaeys, and P. Grangier, Phys. Rev. A 75, 013406 (2007).

16. C. Tuchendler, A. M. Lance, A. Browaeys, Y. R. P. Sortais, and P. Grangier, Phys. Rev. A 78, 033425 (2008).

17. C. Cohen-Tannoudji, J. Dupont-Roc, and G. Grynberg, Photons and Atoms: Basic Process and Applications (Wiley, 1997).

18. The exact origin of the chirp is still under investigation. It might come from a small variation of the residual light shift of the atomic transition frequency during the switching off of the dipole trap laser on this small time scale.

19. We also note that the optical Bloch equations predict a temporal width of the scattered pulse that varies slightly with the detuning [see Fig. 2(b)]. 